\title{
Competitividad del sector industrial de la leche en el estado Zulia- Venezuela
}

\author{
Boscán, Mariby* \\ Sandrea, Maryana* \\ * Economistas. Docentes-Investigadoras de la Universidad del Zulia. Instituto de \\ Investigaciones de la Facultad de Ciencias Económicas y Sociales. Departamento de \\ Estudios Microeconómicos. \\ Cursantes del Programa de Maestría en Gerencia de Empresas. \\ Email: marysandrea@yahoo.com 〜 maribyboscán@yahoo.com.
}

\section{Resumen}

En el presente artículo se analiza la competitividad del sector industrial de la leche en el estado Zulia, con la finalidad de determinar los factores que han estimulado o frenado su desarrollo. La investigación es descriptiva, teniendo como principal fuente de información una entrevista a los gerentes de las empresas del sector, basado en las herramientas teóricas desarrolladas por Michael Porter. Los resultados revelan que las empresas del sector industrial de la leche en el estado Zulia son medianamente competitivas en el ámbito nacional, evidenciando una capacidad ociosa superior al 50\%. En cuanto al análisis de los elementos del diamante competitivo, se pudo apreciar que éstas presentan baja calificación del factor humano, intensa rivalidad y una reducción considerable en la demanda de sus productos. La influencia de la actuación del gobierno no ha sido eficiente, debido a las contradicciones de las políticas aplicadas. Por otro lado, como elemento positivo, las empresas del sector cuentan con una variada gama de industrias relacionadas y de apoyo. Asimismo, en cuanto a las fuerzas competitivas, más del $70 \%$ de los proveedores y consumidores se encuentran en el país, además el sector presenta fuertes barreras de entrada (alta inversión en tecnología) y su producto no tiene sustituto perfecto. Por tanto se puede concluir que, estas empresas no alcanzan el ámbito competitivo internacional, al tiempo que la mayoría de ellas, utilizan estrategias competitivas que se apoyan en la diferenciación para lograr un posicionamiento estratégico a largo plazo.

Palabras clave: Industrial de la leche, competitividad, diamante competitivo, fuerzas competitivas.

\section{Competitiveness in the Milk I ndustry Sector in Zulia State-Venezuela}




\section{Abstract}

In this article the competitiveness of the milk industry sector in Zulia State is analyzed, for the purpose of determining the factors that have stimulated or restrained its development. The research is descriptive, and the main sources of information were interviews made with managers of these companies, based on the theoretical tools developed by Michael Porter. The results reveal that the companies in the milk industry sector in Zulia State are moderately competitive on the national level, demonstrating an idle capacity of over $50 \%$. As far as the analysis of the elements in the competitive diamond, the human factor had a low rating, there is intense rivalry among companies, and a considerable reduction in the demand for their products. The influence of governmental performance has not been efficient, due to the contradictions in the policies applied. On the other hand, as positive elements, the companies in this sector have a varied range of related and support industries. Also, in relation to competitive elements, more than $70 \%$ of the suppliers and consumers are national. In addition the sector presents strong barriers to entrance (high investment in technology) and its products do not have perfect substitutes. Therefore it is possible to concluded that although these companies do not reach the international competitive field, most of them utilize national competitive strategies based on differentiation in order to obtain strategic positioning in the long run.

Key words: Milk industrial, competitivity, competitive diamonds, competitive forces.

Recibido: 02-07-29. Aceptado: 03-06-13

\section{I ntroducción}

En el contexto internacional se han producido una serie de cambios importantes en la última década, que tienen su máxima expresión en el proceso de globalización, lo cual está imponiendo considerables presiones a los países, impulsando la competencia y llevando a alguno de ellos a abrir sus mercados.

Por tanto, la competitividad se ha convertido en un tema de gran importancia para las empresas y gobiernos del mundo, constituyendo uno de los principales factores del comercio internacional. Donde la apertura comercial, los ajustes estructurales y la reconversión productiva, marcan el enfoque competitivo actual (Müller, 1995).

La competitividad según Rosales (1996: 710), es una categoría que se circunscribe a las empresas y cuando se hace referencia a las naciones sólo se puede hacer alusión a la 
noción de productividad. Sin embargo, en la medida en que se reconoce que el territorio tiene condiciones que como tal lo favorecen o desfavorecen, por ejemplo, atrayendo o desechando la inversión extranjera y además influyen sobre el desempeño de las actividades que tienen lugar dentro de él, comienza a ganar sentido hablar de competitividad de una nación o región. En el campo empresarial, es preciso decir que una empresa competitiva es capaz de formular y aplicar estrategias para ampliar o conservar una posición sostenible en el mercado. El desempeño empresarial, es el resultado de la capacitación acumulada previamente, la que a su vez se deriva de estrategias basadas en las percepciones dominantes en las empresas sobre el proceso de competencia y el entorno económico.

El estudio de elementos como los factores de producción, condiciones de la demanda, industrias relacionadas y de apoyo, estructura y rivalidad de las empresas y el rol del gobierno, junto con el análisis de la estructura del sector donde operan las distintas empresas, ayudan a fortalecer la capacidad de las mismas, al permitir la definición de estrategias que contribuyan a mejorar el desempeño competitivo del sector en el ámbito, nacional y regional.

En este sentido, considerando la importancia que tiene a nivel mundial la industria de productos lácteos y su impacto en el desarrollo económico del país, el presente artículo pretende analizar la competitividad del sector industrial de la leche en el estado Zulia, para el periodo 2001-2002.

Para lograr este objetivo, se diseñó un estudio descriptivo, utilizando una entrevista dirigida a los gerentes de las empresas del sector industrial de la leche, registradas en la cámara de industriales del estado Zulia, en total se entrevistaron ocho empresas.

En el presente artículo, se entenderá por sector industrial de la leche, el conjunto conformado por los competidores actuales, competidores potenciales, proveedores, compradores y productos sustitutos de la leche.

Porter (1998), identificó algunos elementos claves para el análisis de la competitividad, los cuales englobó en lo que se conoce como el diamante competitivo, entre los cuales se encuentran: las condiciones de los factores productivos, las condiciones de la demanda, las industrias relacionadas y de apoyo; y la estrategia, estructura y rivalidad de las empresas. Estos elementos adaptados al sector industrial en referencia, más el estudio de las relaciones entre los componentes del mismo (definidas por el autor como las fuerzas competitivas), entre las que destacan: el poder de negociación de los proveedores, el poder de negociación de los compradores, la amenaza de los sustitutos, la amenaza de los 
nuevos ingresos y la intensidad de la rivalidad, permiten estudiar la competitividad del sector industrial de la leche.

Por lo antes expuesto, las variables objeto de estudio comprenden, los elementos del diamante competitivo y las fuerzas competitivas para el sector.

En la investigación realizada se confrontaron considerables limitaciones, entre las que destacan la inexistencia de un registro actualizado de las empresas que conforman el sector industrial de la leche del estado Zulia y la poca receptividad por parte de la gerencia de algunas empresas, por lo cual el marco poblacional definido no es exhaustivo, se considera que el estudio es cualitativo, aunque se utilicen técnicas de análisis cuantitativas, razón por la cual los resultados no serán concluyentes, pero si permitirán revelar tendencias interesantes sobre el comportamiento de las variables estudiadas.

\section{Situación y tendencias del sector industrial de la leche a nivel mundial}

El proceso de globalización de los mercados alimenticios ha sido generado o impulsado por muchos factores, entre los que destacan: el desarrollo de las comunicaciones, el turismo, los movimientos migratorios y la homogeneización de los hábitos de consumo (García, 1998: 18). Diversos cambios están ocurriendo o deben ocurrir en la producción primaria, sobre todo de los países en desarrollo, para que se ajuste al nuevo clima de competitividad y globalización. De forma tal, que existen grandes riesgos sino se logran armonizar las estrategias de modernización de la producción primaria con las introducidas por los acuerdos internacionales y la comercialización. Por lo cual, la solidez y prosperidad de las economías de los que hoy son países desarrollados, tiene como elemento importante y responsable de ese desarrollo el crecimiento y el progreso acelerado de su sector agrícola.

De los productos agrícolas que se producen a nivel mundial la leche y sus derivados representan aproximadamente 524 millones de toneladas métricas (TM) al año. De ese total solamente el $5 \%$ se comercializa internacionalmente lo que significa que el mercado de estos productos es básicamente nacional y lo que se comercializa en el mercado mundial es una cantidad residual, aunque esta situación varía dependiendo del país (Garzon, 1996: 6).

Según la Cámara Venezolana de Pasteurizadoras (CAVEPAS) (1998: 17), las excepciones a esta regla, la constituye por el lado de los exportadores, Nueva Zelanda y Australia con un $90 \%$ y $45 \%$ respectivamente exportable de su producción. Del lado de los importadores de leche, Venezuela es una de las excepciones a nivel mundial puesto que 
sus importaciones tradicionales han llegado a representar aproximadamente un $50 \%$ del consumo de leche en polvo.

\section{Características generales del sector industrial de la leche en el ámbito nacional}

La apertura y globalización en Venezuela se han interpretado como un derrumbamiento total de las barreras de comercio, sin el menor criterio de la importancia que tiene la dependencia y vulnerabilidad alimentaría a la que se expone el país. Competir con los productos ḱcteos de alta tecnología y amplia protección, como los de la Comunidad Europea, Nueva Zelanda, Australia, Canadá y los Estados Unidos, sería una tarea difícil, sino imposible, para los productores venezolanos, lo cual parece indicar que será el sector del comercio importador y no el productor, quien obtenga las mejores ventajas competitivas.

En este sentido, la producción láctea constituye uno de los sectores en Venezuela que presenta baja productividad en comparación con promedios internacionales, productividad reflejada en rendimientos por vaca con un promedio de 4 litros/día, aunque algunos productores obtienen más de 10 litros/día, cuando la media internacional esta sobre 18 litros por día (Coello, 1998: 11).

Para el año 1989 la producción nacional de leche cruda alcanzó un volumen de 1.638.725 miles de litros; en tanto que en 1998, se ubicó en 1.440.230 miles de litros, lo cual indica una tasa de crecimiento interanual promedio de $-12.11 \%$, para el período de 1989-1998, debido a los cambios producidos en la economía nacional y al proceso de implantación de las políticas de estabilización y ajuste.

Con el cierre de las instituciones financieras en 1994, el sector perdió gran parte de su músculo inversor, además de una fuente de préstamos preferenciales (Ver Tabla 1). 
Tabla 1

Producción de leche cruda en Venezuela (miles de litros)

\begin{tabular}{ccc}
\hline Años & Producción & Tasa de crecimiento interanual (\%) \\
\hline 1989 & 1.638 .725 & $-11,38$ \\
1990 & 1.452 .227 & 0,63 \\
1991 & 1.461 .428 & 6,38 \\
1992 & 1.554 .648 & 6,90 \\
1993 & 1.661 .919 & $-19,13$ \\
1994 & 1.344 .051 & 2,00 \\
1995 & 1.370 .932 & 2,50 \\
1996 & 1.405 .205 & 3,72 \\
1997 & 1.457 .472 & $-1,18$ \\
1998 & 1.440 .230 &
\end{tabular}

En lo referente al consumo aparente total de leche, se evidencia una diferencia entre la demanda y la oferta nacional, es decir entre el consumo de leche y la producción. Este déficit en la producción de leche durante la última década, es lo que ha llevado a Venezuela a ser un importante demandante de leche en el mercado internacional (Ver Gráfico 1).

Esta importación ha sido básicamente de leche en polvo, a granel, la cual es envasada en el país. La tendencia de consumo nacional ha ido acorde con la oferta internacional, aumentando el consumo de leche entera en la última década, por cuanto se ha evidenciado un cambio en el patrón de consumo de leche del venezolano, el cual puede explicarse por la pérdida de poder adquisitivo del consumidor, dado que la leche pasteurizada es más costosa que la pulverizada (Garzón, 1996: 22). Por otro lado si se toma en cuenta el consumo aparente por persona, se puede notar que en Venezuela, éste ha sido inferior al consumo mínimo normativo, según la organización de las Naciones Unidas para la Agricultura y la Alimentación (FAO) (1997: 46) de 130 litros/habitantes/año (L/H/A), fluctuando entre un valor mínimo de 80,59 L/H/A en 1994, hasta un máximo de 108,86 L/H/A en 1991. La reducción abrupta del consumo per-cápita de leche en todas sus formas es la expresión negativa más relevante del deterioro progresivo de la calidad de la dieta de los venezolanos. 
Gráfico 1

Producción y Consumo Nacional de Leche Cruda

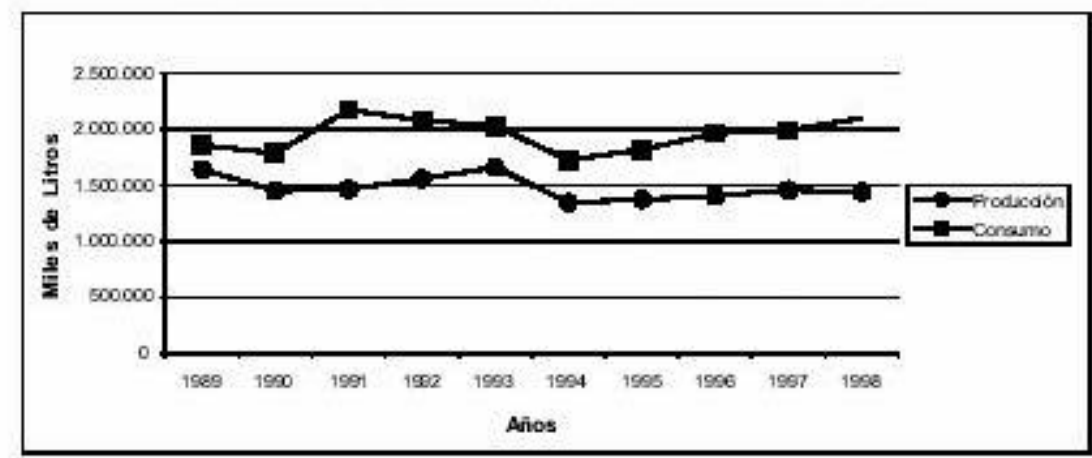

Fuente: M.P.C (1998).

Resulta evidente para todos los involucrados en el sector industrial de la leche, la necesidad de un gran esfuerzo nacional, para promocionar el consumo de la leche en todas sus formas y muy especialmente el consumo de leche fresca. Los programas de asistencia alimentaria que tienen como base el suministro gratuito, o a precios reducidos, para la población vulnerable, han contado con el apoyo gubernamental, a ún en situaciones económicas y sociales muy difíciles. En general estos programas han tenido éxito porque inciden directamente en el consumidor final.

Durante los últimos años, en términos de consumo per-cápita, Venezuela ha alcanzó un promedio de 92,65 L/H/A, estando sólo por encima de países que tradicionalmente han tenido problemas agudos de nutrición, como Haití (30 L/H/A), Bolivia (55 L/H/A) y Perú (60 L/H/A), según cifras de la asociación de industriales de leche en polvo (ASOLEP) (1996: 35). Esta situación se vio agravada por la decisión gubernamental de monetizar los programas sociales, al sustituir la beca láctea que suministraba un aporte parcial en el consumo de leche para la población de menores recursos, por un pago en dinero que se desviaba hacia otros fines.

\section{Caracterización y situación del sector industrial de la leche en el estado Zulia}

Según las consideraciones del Instituto de Estudios Superiores de Administración (IESA) (1997: 171), una de las principales transformaciones que predominan en el mundo actual es la relevancia que ha adquirido el desarrollo de regiones particulares dentro de cada país. Más aún se afirma, que las regiones son los espacios idóneos para desarrollar la competitividad, mucho más que la nación, debido a que el análisis en el ámbito nacional, esconde diferencias regionales en factores tales como mano de obra, tecnología e infraestructura, así como en la estructura de la demanda y la rivalidad entre las empresas. 
El estado Zulia es una de estas regiones, siendo el primer productor de leche del país, ya que aporta para 1998 más del 35\% de la producción nacional y conjuntamente con los estados Falcón, Táchira y Mérida (cuenca del lago de Maracaibo), proporcionan el 59,92\% de la misma, esta situación ha contribuido a la relevancia del impacto de este sector en la actividad económica, dadas las dimensiones que el mismo tiene en el país. La concentración espacial alrededor del Zulia ha determinado tanto los esquemas de producción (ganado de doble propósito), como los de consumo (leche en polvo), por lo cual, el sector industrial de la leche es considerado motor potencial para el desarrollo de la región zuliana.

Para describir de forma más detallada la situación del sector industrial de la leche en el estado Zulia, se realizaron un total de ocho entrevistas a los gerentes de las empresas productoras y transformadoras de leche, registradas en la cámara de industriales del estado Zulia. Puede observarse que, el número de industrias no es representativo de la población total existente en el estado Zulia, sin embargo los resultados que se muestran a continuación reflejan ciertas tendencias sobre el comportamiento de las variables estudiadas.

Según los resultados obtenidos a partir de las entrevistas efectuadas, las empresas del sector industrial de la leche del estado Zulia, pertenecen en un $75 \%$ de los casos analizados a la pequeña y mediana industria, es decir, están conformadas por cinco hasta cien personas (considerando la clasificación de la oficina central de estadística e información), por lo tanto, el 25\% corresponde a la gran industria, la cual le proporciona empleo a más de cien personas.

En lo que respecta al margen de capacidad ociosa, la muestra analizada revela que existen dos empresas, es decir, un $25 \%$ con un comportamiento atípico (inexistencia de capacidad ociosa). Sin embargo, el $75 \%$ de las empresas estudiadas (la mayor parte de la muestra), evidencia un nivel de capacidad ociosa que iguala o supera el $50 \%$, por lo tanto, su nivel competitivo es bajo al menos en cuanto al análisis de éste elemento, dejando abierta la posibilidad para las empresas del sector, de buscar estrategias que les permitan incursionar en nuevos mercados en el ámbito regional, nacional e internacional, y así poder utilizar al máximo su capacidad instalada.

En referencia a la opinión gerencial sobre el nivel competitivo, la encuesta realizada reveló que el $87,5 \%$ de los gerentes afirmaron que su producto Íder es competitivo, de los cuales el $75 \%$ consideró que el nivel de competitividad del mismo era alto.

Considerando la capacidad real de producción y la opinión de los empresarios sobre el 
nivel competitivo, puede decirse que en el ámbito nacional el sector industrial de la leche es medianamente competitivo.

Los gerentes entrevistados consideraron que las fuentes potenciales de ventajas competitivas en el sector, están centradas en los siguientes elementos: calidad del producto final $(37,5 \%)$, cadena de precios $(12,5 \%)$, marca por tradición $(12,5 \%)$, el resto $(37,5 \%)$ no respondió.

Los resultados de las entrevistas realizadas reflejan que el 62,5\% de las empresas del sector industrial de la leche del estado Zulia, utilizan en forma individual la diferenciación de sus productos como un elemento clave para el diseño de su estrategia competitiva, el costo en un $12,5 \%$, mientras que un $25 \%$ de los gerentes nunca han elaborado estrategias competitivas.

Es importante resaltar, que el hecho de que la mayor parte de los gerentes encuestados elaboren estrategias competitivas $(62,5 \%)$, es un aspecto favorable que podría ayudar a incrementar la competitividad del sector en el ámbito nacional e internacional.

Con respecto a los factores internos que frenan el desarrollo de las empresas, se encontró que un $37,5 \%$ de los gerentes encuestados manifestó como factor individual la insuficiencia de maquinarias y equipo, otro $37,5 \%$ la adaptación de equipos y maquinarias, destacándolos como los principales problemas que afectan el nivel competitivo de las empresas. También, un $12,5 \%$ consideró los problemas de administración de costos y mercadeo, dentro de los elementos que afectan la competitividad y el resto $(12,5 \%)$ no respondi ó.

En relación con los factores externos, cabe destacar que el $75 \%$ de los gerentes encuestados, no identificaron un problema individual que afecte su competitividad, sino que afirmaron que existe una combinación de factores que frena su desarrollo, entre los cuales destacan: la competencia desleal (el contrabando), problemas de financiamiento, la ineficacia de políticas oficiales para impulsar el desarrollo del sector e insuficiencia de demanda, la cual en forma individual arrojó el $25 \%$.

\section{Diamante competitivo}

El análisis del contexto de la competencia estaría incompleto si no se consideran los cuatro determinantes o atributos genéricos, que conforman el entorno en el que tienen que competir las empresas locales, y que fomentan o entorpecen la creación de la ventaja competitiva. 
Los determinantes de la ventaja nacional, como son conocidos en la metodología de Porter (1998), se encuentran resumidos en una herramienta que él mismo ha denominado el diamante competitivo. A través del cual es posible entender el por qué un sector específico de una nación podrá alcanzar el éxito frente a sus rivales internacionales.

Los cuatro determinantes o atributos genéricos son los siguientes: condiciones de los factores productivos, condiciones de la demanda, industrias relacionadas y de apoyo (el cluster) y la estrategia, estructura y rivalidad de las empresas. Es importante destacar además, un quinto elemento que aunque no es considerado como atributo genérico, debe ser analizado por su importancia en los procesos de competitividad a nivel nacional e internacional, el mismo se refiere a la posición o papel del gobierno.

\subsection{Condiciones de los factores productivos}

El bajo nivel de calificación del trabajador ha sido un freno para el desarrollo tecnológico del sector productor de leche. Los procesos son intensivos en mano de obra. En la industria procesadora la situación es diferente: hay plantas modernas con gran capacidad de envasado y tecnología para el procesamiento, en tanto que la vialidad requiere mayor mantenimiento y en general los servicios públicos son ineficientes.

La mano de obra es tradicionalmente de origen colombiano, dispuesta a aceptar las condiciones de trabajo y salario mínimo. El bajo nivel de capacitación del personal limita el uso de tecnologías tales como el ordeño mecánico, en la fase de producción primaria de leche, también influye el poco interés por parte del productor para entrenar a los trabajadores. Por otra parte, la inestabilidad económica y el deterioro del salario real, están ocasionando escasez de personal en esta fase. Mientras que, en la agroindustria aunque no existe escasez de personal, el nivel de instrucción es insuficiente, incluso en el nivel gerencial, aunque muchos gerentes poseen amplia experiencia.

\subsection{Condiciones de la demanda}

Antes de 1989, debido a la política de sustitución de importaciones y las políticas de control de cambio, la industria local tenía una demanda asegurada, pues no existía la opción de elegir entre lo local y lo importado.

Luego de la apertura comercial y las medidas de ajuste adoptadas en el país para 1989, el consumo de ácteos y derivados disminuyó. Según cifras presentadas por CAVEPAS (1997: 6), el consumo per-cápita de leche en Venezuela pasó de 109,22 L/H/A en 1991 a 85,19 L/H/A en 1997, debido a la baja en el poder adquisitivo del venezolano, sin 
embargo, la producción de las empresas se ha mantenido ya que todos sus productos son colocados en el mercado nacional.

Se debe resaltar, que Venezuela ha sido tradicionalmente un importador de productos lácteos. Sin embargo, existe cierta exportación de leche condensada, leche de larga duración y de chicha.

El gobierno, también ha ejercido influencia importante en la demanda de productos lácteos como parte de sus programas sociales, tal es el caso de la leche para el escolar y las mujeres embarazadas.

\subsection{Industrias relacionadas y de apoyo}

La existencia en el país de industrias relacionadas y de apoyo (cluster) internacionalmente exitosas, conduce a la generación de ventajas competitivas porque permite el acceso económico, eficiente, rápido y en algunos casos, preferencias sobre determinados insumos, asimismo, cooperación en procesos de innovación y mejora, distribución, marketing o servicio al cliente, entre otros.

Entre las industrias relacionadas y de apoyo se encuentran: centros de inseminación artificial (empresas Bull Semen y Viateca), centros de genética y recría dedicados a la selección de razas, laboratorios dedicados a la fabricación de productos veterinarios (Calox, Pfizer, Cooper y otros), transportistas independientes, comercializadores de maquinarias, empresas (ntersan, Ferrelago y Ford), fabricantes de envases de hojalata para la leche en polvo (Domínguez y Compañía y Envases Venezolanos); Tetra Pack y Envases Internacionales son las únicas proveedoras de maquinarias y material de envasado para el subsector de leche de larga duración (UHT) y Pequiven provee el material para los envases de plástico rígido y las procesadoras los elaboran en sus plantas.

\subsection{Estrategia, estructura y rivalidad de las empresas}

En el sector industrial de la leche, es posible evidenciar un elevado grado de fragmentación, lo que aunado a las importaciones provoca que exista una intensa rivalidad. Con la apertura comercial, impulsada en 1989, la liberación de precios y la disminución de los aranceles de importación, las empresas se han visto en la necesidad de competir con empresas extranjeras que entran al mercado nacional.

La mayoría de las empresas están constituidas por más de 30 trabajadores y su 
estructura está fuertemente atomizada. Además, existe una elevada diversificación de productos, asociado con una alta creatividad y monitoreo de las exigencias del entorno, aspectos que constituyen ventajas para el sector.

\subsection{Posición del gobierno}

Según Porter (1998: 21), el papel real de los gobiernos en la consecución de la ventaja competitiva está en influenciar a los otros determinantes. No cabe duda, que el gobierno venezolano ha jugado diferentes roles en el desarrollo del sector industrial de la leche.

La política implantada por el gobierno en los últimos años, ha sido inestable, en algunos casos se apoya la producción de carne y en otros la de leche. Esta política ha incrementado la variabilidad de los cruces de razas e impedido el desarrollo de una selección genética especializada, ha desestimulado la inversión privada y el aprendizaje continuo que permitirían desarrollar la competitividad del sector. Los controles han creado incertidumbre en cuanto al precio final del producto, el costo de los insumos y los aranceles de importación de leche en polvo. Por lo cual, la producción de leche se ha convertido en un negocio de alto riesgo.

\section{Fuerzas competitivas del sector industrial de la leche en el estado Zulia}

En el estudio de la competitividad, Porter (1998) afirma, que es importante analizar la estructura del sector en la cual compite la empresa. Este análisis estructural parte de considerar al sector industrial como el entorno ambiental donde la empresa interactúa con las demás firmas competidoras.

Además supone que en cualquier sector, las reglas de la competencia están englobadas en cinco fuerzas competitivas, y las mismas permiten determinar la rentabilidad de la industria, porque influyen en forma directa sobre los precios que puede cobrar una empresa, su estructura de costos y sus requerimientos de inversión (Porter, 1998: 22).

\subsection{Poder de Negociación de los Proveedores}

Las entrevistas realizadas permitieron determinar un elevado grado de diversidad en la procedencia de los proveedores de éste sector, debido a que el $75 \%$ se encuentran localizados tanto en el territorio nacional como en el exterior y sólo el $25 \%$ de los gerentes consultados respondieron que sus proveedores se ubicaban a nivel regional.

Entre los principales proveedores del sector lácteo del estado Zulia y de sus productos 
líderes destacan: leche cruda (hacienda El Capitán, hacienda El Milagro, Agropecuaria Monte Mario, Masfinca, Canprolac, Joosten Products y otras fincas), leche en polvo (New Zeeland Milk Products), concentrados de jugos (Izaza y New Zeland Milk Products), cremas $40 \%$ de grasa (Parmalat y Genica), químicos (Productora Química Industrial), envases (Envases Internacional, Inversiones Selva y Nacional Plastic), etiquetas (Disza), material de oficina (Nebabrica y otros) y servicios aduanales (Fritz de Venezuela).

Con relación a los problemas que confrontan las empresas del sector industrial de la leche del estado Zulia con sus proveedores, se evidenció que el 87,5\% afirmó no presentar dificultades y el resto $(12,5 \%)$ no respondió.

\subsection{Poder de Negociación de los Compradores}

Los compradores o clientes del sector industrial de la leche del estado Zulia, se encuentran localizados aproximadamente en un $90 \%$ en el territorio nacional, del cual un $40 \%$ están ubicados en el mencionado estado. Siendo entonces, determinante para los empresarios maracaiberos, el estudio de las costumbres, valores, condiciones climáticas y condiciones demográficas del estado, al momento de fabricar sus productos.

\subsection{Barreras de entrada}

Se pudo conocer durante la interacción generada por las entrevistas, que en este tipo de industrias existen importantes barreras, ya que se requiere de una alta inversión en tecnología (sobre todo en las industrias Pasteurizadoras y pulverizadoras), debido a que el sector es intensivo en capital y también se necesitan altos volúmenes de capital de trabajo.

\subsection{Productos sustitutos y relacionados}

En relación a esta fuerza competitiva, es de hacer notar que, la leche es un producto de primera necesidad que no tiene sustituto perfecto, sin embargo, según las opiniones de los gerentes consultados, los productos líderes en particular, tienen productos sustitutos en el mercado interno, entendiendo o considerando como sustitutos otras marcas de leche (pasteurizada y pulverizada) diferentes a las que ellos ofrecen, las cuales pueden ser nacionales o importadas.

\subsection{Estructura de la Competencia}

En los actuales momentos, producto de la crisis económica y social que confronta el país, 
se ha generado una intensa rivalidad entre los competidores, debido especialmente a la entrada de leche importada (que en algunas ocasiones pueden ser ilegales o sin control sanitario), de mejor calidad y a más bajo precio.

Las empresas, por tanto, deberán basar su estrategia de competencia en la diversificación del producto, con una minimización de los costos, donde el precio no es el elemento central de la competencia, para así lograr un posicionamiento estrat égico a largo plazo.

\section{Consideraciones finales}

El desarrollo de capacidades para competir y más concretamente la creación y/o identificación de ventajas competitivas, se han convertido en la actualidad en elementos claves para tener éxito en la penetración de mercados. En éste sentido, el $87,5 \%$ de los gerentes encuestados consideraron que su producto líder era competitivo y además, de éstos un $75 \%$ afirmaron que el grado de competitividad era alto, en el ámbito nacional. Sin embargo, a nivel internacional estas empresas no son competitivas, debido fundamentalmente a la baja productividad, medida a través de los rendimientos por vaca. Así mismo, el 75\% de las empresas estudiadas evidenciaron una capacidad ociosa igual o superior al $50 \%$. Por lo que se concluye que estas empresas a nivel nacional presentan un nivel de competitividad medio.

Algunos de los factores internos que afectan negativamente el desarrollo de la capacidad competitiva de este sector industrial, están relacionados con la insuficiencia de maquinarias y equipos (37,5\% de los gerentes encuestados), adaptación de equipos y maquinarias $(37,5 \%)$ y problemas de administración de costos y mercadeo $(12,5 \%)$.

Mientras que, el $75 \%$ de los gerentes entrevistados consider ó que los factores externos debían ser evaluados en forma conjunta y no individual, destacando entre ellos la competencia desleal, problemas de financiamiento, ineficiencia de políticas oficiales e insuficiencia de demanda del producto.

El estudio de los elementos del diamante competitivo, permitió constatar dentro de los factores productivos, un bajo nivel de capacitación del personal, lo cual limita el uso de tecnología avanzada. Por otra parte, la inestabilidad económica y el deterioro del salario real, están ocasionando escasez de personal en el sector industrial de la leche.

Además pudo evidenciarse, en las condiciones de la demanda, que luego de la apertura comercial y las medidas de ajuste adoptadas en el país para 1989, el consumo de lácteos 
y derivados disminuyó, debido a la baja en el poder adquisitivo del venezolano. En cuanto a las industrias relacionadas y de apoyo, estas constituyen una importante fuente de ventajas competitivas, ya que el sector dispone de una gran cantidad de empresas de apoyo que ofrecen servicios al productor y a la industria procesadora.

Cabe destacar, que el sector industrial de la leche presenta una intensa rivalidad. Así mismo, la mayoría de las empresas están constituidas por más de 30 trabajadores y su estructura está fuertemente atomizada.

En cuanto al rol del gobierno venezolano, éste ha sido principalmente, como controlador de precio, influyendo negativamente la inconsistencia de las políticas oficiales sobre el nivel competitivo del sector.

Al analizar los componentes de las fuerzas competitivas, del sector industrial de la leche del estado Zulia, pudo determinarse que los proveedores se encuentran localizados tanto a nivel nacional como internacional (75\%), lo cual conlleva a que se elaboren constantemente estrategias de negociación aguas arriba.

Con relación a los consumidores, los mismos ejercen poder debido a que se concentran en más del $90 \%$ en el territorio nacional ( $40 \%$ en el estado Zulia). El consumidor maracaibero, presenta preferencias hacia el consumo importado, lo cual presiona a los gerentes a ser más competitivos y mejorar la calidad de sus productos.

Con la investigación pudo conocerse que existen importantes barreras de entradas al sector, ya que se requiere de una alta inversión en tecnología y volúmenes de capital de trabajo, asimismo, se evidencio que la sustitución de la leche se daba sólo en términos de una marca por otra, o de un estado físico-químico por otro (leche en polvo-leche líquida o leche pasteurizada normal-leche de larga duración, entre otros).

Las empresas, por lo tanto, deberán basar su estrategia de competencia, para lograr un posicionamiento estratégico a largo plazo, en la diversificación del producto, con una minimización de los costos, donde el precio no sea el elemento central de la competencia.

\section{Referencias Bibliográficas}

1. Asociación de Industriales de Leche en Polvo (ASOLEP) (1996), Análisis de la problemática del sector lácteo. Caracas-Venezuela.

2. Cámara Venezolana de Pasteurizadoras (CAVEPAS) (1997), Informe sobre la 
situación de la industria pasteurizadora. Caracas-Venezuela.

3. Cámara Venezolana de Pasteurizadoras (CAVEPAS) (1998), Circuito lácteo venezolano. Anuario estadístico. Caracas-Venezuela.

4. Coello, León R. (1998), Estudio de competitividad de la empresa Industria de lácteos La Fe, C.A. (I LAFECA) dentro del circuito lácteo nacional. Trabajo para optar al titulo de magíster. Caracas-Venezuela. Universidad Central de Venezuela.

5. García Álvarez Coque, J. M. (1998), Globalización y comercio mundial de productos agroalimentarios. En Simposio internacional globalización y sistemas agroalimentarios. Caracas- Venezuela. Fundación Polar.

6. Garzón, Blanca (1996), Sector: productos lácteos. Volumen III, forma parte del proyecto Zulia: competitividad para el desarrollo. Caracas-Venezuela. Ediciones IESA. Centro de gerencia estratégica y competitividad.

7. Instituto de Estudios Superiores de Administración (IESA) (1997). Zulia: competitiva para el desarrollo. Estudios de competitividad regional. CaracasVenezuela. Ediciones IESA, C.A.

8. Ministerio de Agricultura y Cría (1998), Estadísticas Pecuarias. Caracas-Venezuela.

9. Ministerio de Producción y Comercio (1998), Dirección General de Cadenas Agropecuarias Caracas-Venezuela.

10. Müller, Geraldo (1995), El Caleidoscopio de la competitividad. Revista de la CEPAL No 52. Agosto.

11. Organización de las Naciones Unidas para la Agricultura y la Alimentación (FAO). (1997), Situación y perspectiva de los productos básicos. Caracas-Venezuela.

12. Porter, Michael E. (1998), Ventaja competitiva: creación y sostenimiento de un desempeño superior. México. Primera Edición: 1987. décima Sexta Reimpresión. Compañía Editorial Continental. S.A. de C.V. Titulo original: Competitive advantage: creating and sustaining superior performance. Traducción: María Ascensión de la Campa Pérez Sevillag.

13. Rosales, Ramón (1996), Estrategias gerenciales para la pequeña y mediana 
empresa. Caracas-Venezuela. Ediciones IESA. Fundación Polar. Editorial Ex Libris. P.p.131. 